Provided for non-commercial research and education use. Not for reproduction, distribution or commercial use.

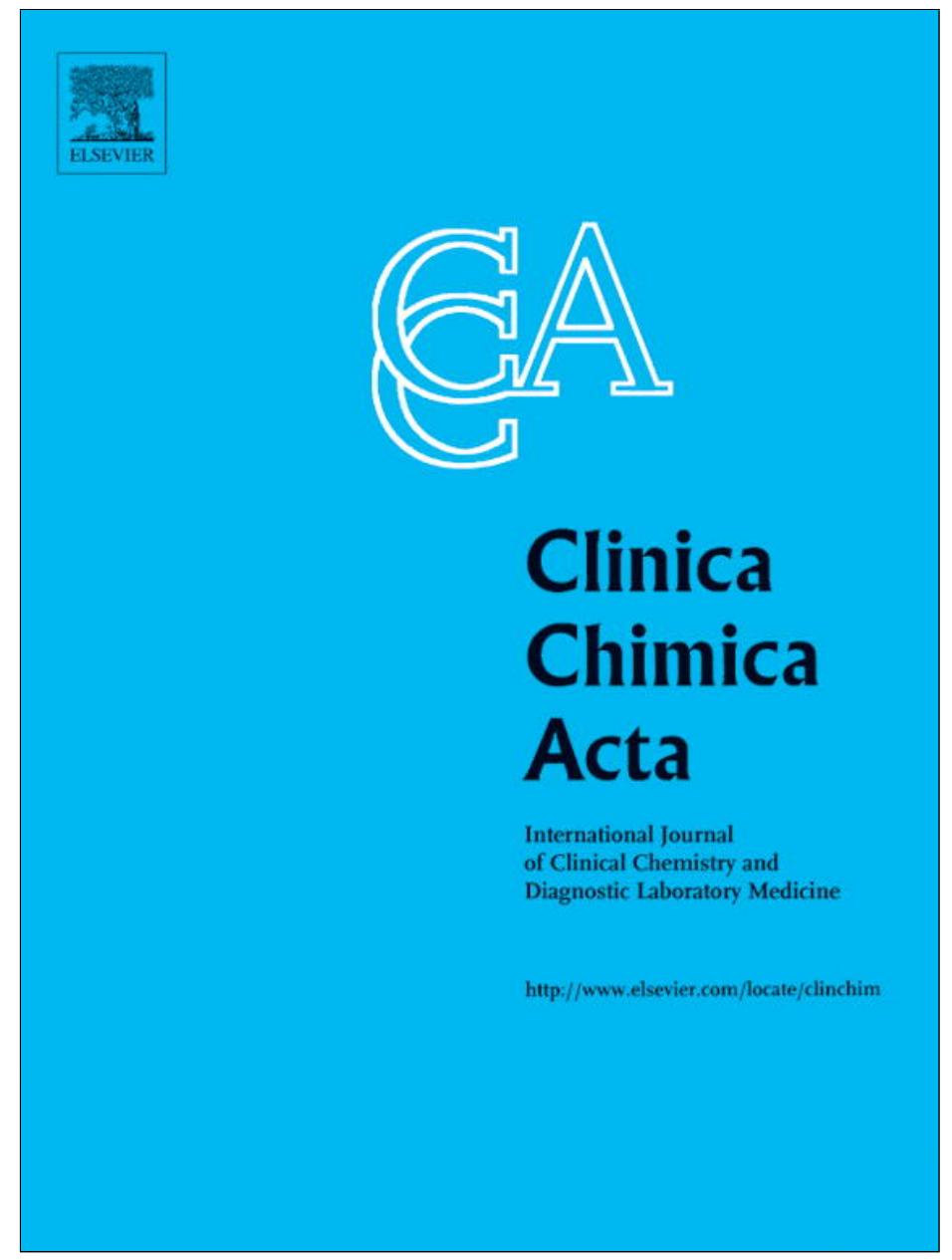

(This is a sample cover image for this issue. The actual cover is not yet available at this time.)

This article appeared in a journal published by Elsevier. The attached copy is furnished to the author for internal non-commercial research and education use, including for instruction at the authors institution and sharing with colleagues.

Other uses, including reproduction and distribution, or selling or licensing copies, or posting to personal, institutional or third party websites are prohibited.

In most cases authors are permitted to post their version of the article (e.g. in Word or Tex form) to their personal website or institutional repository. Authors requiring further information regarding Elsevier's archiving and manuscript policies are encouraged to visit:

http://www.elsevier.com/copyright 
Letter to the Editor

\section{Evaluation of analytical performance of a novel immunoenzymometric assay for cTnI}

\section{Keywords:}

cTnI

cTnT

Healthy subjects

Cardiac markers

Reference values

\section{To the Editor}

We evaluated the analytical performance of the immunoenzymometric assay for the cTnI, named ST AIA-PACK cTnI 3rd-Gen, using the automated AIA-2000 platform (Tosoh Corporation, Tokyo, Japan). This method is a two-site immunoenzymometric assay, which uses a combination of two monoclonal antibodies, respectively directed to 41-49 and 87-91 amino acids of the cTnI peptide chain, and the ternary troponin ITC complex as a calibration antigen [1].

Blood samples from patients with cardiac diseases or healthy subjects were collected in polypropylene tubes without or with lithium heparin. Samples were then rapidly centrifuged at $3000 \times g$ for $10 \mathrm{~min}$ and plasma was then analyzed within $1 \mathrm{~h}$ or alternatively, stored at $-80^{\circ} \mathrm{C}$.

The limits of blank (LoB) and detection (LoD) for cTnI assay were determined according to the CLSI EP17-A protocol [2]; the calculated LoB and LoD values were $3.5 \mathrm{ng} / \mathrm{L}$ and $8.7 \mathrm{ng} / \mathrm{L}$, respectively. The assay reproducibility was evaluated in accordance with the CLSI EP5-A2 protocol [3] by repeatedly measuring 3 heparinized plasma samples for consecutive 20 working days with different cTnI concentrations; the results are reported in Table 1 . The between-runs imprecision profile was performed by repeatedly measuring in 20 different runs 10 heparinized blood samples collected from healthy subjects and patients with acute myocardial infarction (mean cTnI concentrations from 13 to $17,782 \mathrm{ng} / \mathrm{L}$ ). The calculated limits of quantitation (LoQ) at $20 \% \mathrm{CV}$ and $10 \% \mathrm{CV}$ were 30 and $100 \mathrm{ng} / \mathrm{L}$ of CTnI, respectively.

We also evaluated whether the measurement in heparinized plasma and serum samples actually gives similar results. A very close linear relationship was found between the cTnI values measured in 105 serum (X-axis) and heparinized plasma (Y-axis) samples of healthy subjects and patients with cardiac diseases (cTnI plasma $=3.10+0.95 \mathrm{cTnI}$ serum; $R=0.999)$. A very close linear regression $(R=0.991, n=521$, $\mathrm{y}=58.3+0.602 \mathrm{x})$ was found between the cTnI values measured with the Tosoh method (Y-axis) and Access AccuTnI method (X-axis), using the UniCell DxI 800 platform (Beckman Coulter, Inc., Fullerton, USA) in 521 heparinized plasma samples of healthy subjects and cardiac patients (Supplemental File 1). However, Tosoh method showed a mean negative bias (i.e., underestimation) of cTnI value compared to BeckmanCoulter by $-30.2 \%(p=0.0024$ by Wilcoxon signed rank test). A close correlation $(\mathrm{R}=0.938, \mathrm{n}=521, \mathrm{y}=-1067+11.6 \mathrm{x})$ was also found between the cTnI and cTnT values, measured with Tosoh method
(X-axis) and Troponin T-hs method (Y-axis), using the automated Cobas e411 platform (Roche Diagnostics, Germany) (Supplemental File 2).

The distribution of cTnI values, measured by the Tosoh method, was evaluated in 452 healthy individuals (M 326, F 126; mean age 45.6 years, median 45 years, interquartile range $33.0-58.0$ years, range 17-76 years), recruited from laboratory staff, blood donors, or voluntary subjects, included in screening programs for preventive medicine. The presence of cardiac or systemic acute or chronic diseases was excluded in all subjects by history, accurate clinical examination, ECG, cardiac imaging, and laboratory tests. Furthermore, all subjects denied the use of drugs for at least two weeks before the sample collection for cTnI assay. The informed consent was obtained by all subjects enrolled in the study and the screening programs were approved by the local ethical committee. The calculated 99th percentile of the cTnI values was $33 \mathrm{ng} / \mathrm{L}$ (median $9.0 \mathrm{ng} / \mathrm{L}$, range $0-44 \mathrm{ng} / \mathrm{L}$ ). The percentages of healthy population with values equal or less than LOB (3.5 ng/L) and LOD (8.7 ng/L) values were $15.7 \%$ and $49.8 \%$, respectively.

Quality specifications [4,5] for troponin measurement recommend an assay imprecision $\leq 10 \% \mathrm{CV}$ for the values corresponding to the 99th percentile of reference population. Use of troponin assays with intermediate imprecision (from $10 \%$ to $20 \% \mathrm{CV}$ ) at the 99 th percentile, however, does not lead to significant patient misclassification, when interpreting serial troponin results $[4,5]$.

Compared to previous second-generation AIA-Pack assay [4], the third-generation AIA-Pack assay for CTnI showed an improved analytical sensitivity and reproducibility, especially at very low cTnI concentrations. Indeed, the LoD and 10\% LoQ values of the second-generation assay, as reported in a previous study [1], were $38 \mathrm{ng} / \mathrm{L}$ and $130 \mathrm{ng} / \mathrm{L}$, respectively, while the corresponding values of the third-generation assay, as assessed in the present study, were $8.7 \mathrm{ng} / \mathrm{L}$ and $100 \mathrm{ng} / \mathrm{L}$. This improved imprecision at lower cTnI concentrations allows the measurement of the 99th percentile of reference population (i.e., $33 \mathrm{ng} / \mathrm{L}$ ) with an imprecision less than $20 \%$, as well as the detection with confidence (measured values above the LoD) of cTnI circulating levels in more than half of normal subjects. These analytical performances suggest that the third-generation AIA-Pack Tosoh assay for cTnI should be considered as "clinically acceptable", according to the scorecard proposed by Apple [4].

The third-generation AIA-Pack Tosoh assay for CTnI showed a very close agreement throughout all the working range with the Access AccuTnI Beckam-Coulter method, used for the routine measurement of cTnI in the Authors' laboratory (Supplemental File 1). Moreover, a

Table 1

Evaluation of assay reproducibility according to the CLSI EP5-A2 protocol.

\begin{tabular}{lllll}
\hline Sample & $\mathrm{n}$ & Mean cTnI concentration (ng/L) & Within-run CV (\%) & Total CV (\%) \\
\hline A & 20 & 22 & 19.62 & 35.46 \\
B & 20 & 52 & 9.14 & 18.42 \\
C & 20 & 119 & 4.00 & 6.50 \\
\hline
\end{tabular}


close agreement was also demonstrated between the cTnI measured by Tosoh methods and those of cTnT measured with ECLIA Roche method (Supplemental File 2). These data suggest that the thirdgeneration AIA-Pack assay is suitable for the clinical evaluation of patients with cardiac diseases.

\section{Sponsorship}

Tosoh Europe N.V. (Tessenderlo, Belgium) kindly supplied all reagents and calibrators used in the study for the measurement of cTni with AIA-1000 platform.

Supplementary data to this article can be found online at http:// dx.doi.org/10.1016/j.cca.2012.11.018.

\section{References}

[1] Pagani F, Stefini F, Toppino M, et al. Multicenter evaluation of the TOSOH AIA-Pack second-generation cardiac troponin I assay. Clin Chem 2004;50:1707-9.

[2] CLSI EP17-A protocol. Protocols for determination of limits of detection and limits of quantitation; approved guidelines, vol. 24, No. 34; 2004 [Wayne, Pennsylvania].

[3] CLSI EP5-A2 protocol. Evaluation of precision performance of quantitative measurement methods, vol. 24, No. 25. Approved guideline, Second edition; 2004 [Wayne, Pennsylvania].

[4] Apple FS. A new season for cardiac troponin assays: it's time to keep a scorecard. Clin Chem 2009;55:1303-6.

[5] Apple FS, Collinson PO. IFCC Task Force on Clinical Applications of Cardiac Biomarkers. Analytical characteristics of high-sensitivity cardiac troponin assays. Clin Chem 2012;58:54-61.
Maria Franzini

Fondazione Toscana G. Monasterio, Pisa, Italy Scuola Superiore Sant'Anna, Pisa, Italy

Concetta Prontera

Silvia Masotti

Fondazione Toscana G. Monasterio, Pisa, Italy

Giancarlo Zucchelli QualiMedLab, Pisa, Italy

Simona Storti

Fondazione Toscana G. Monasterio, Pisa, Italy

Claudio Passino

Aldo Clerico*

Fondazione Toscana G. Monasterio, Pisa, Italy

Scuola Superiore Sant'Anna, Pisa, Italy

*Corresponding author at: Department of Laboratory Medicine, Fondazione CNR-Regione Toscana G. Monasterio,

Scuola Superiore Sant'Anna, Via Trieste 41, 56126 Pisa, Italy.

Tel.: + 390585 493569; fax: + 390585493601.

E-mail address: clerico@ifc.cnr.it (A. Clerico).

2 November 2012 